\title{
Billroth II with Braun Enteroenterostomy Is a Good Alternative Reconstruction to Roux-en-Y Gastrojejunostomy in Laparoscopic Distal Gastrectomy
}

\author{
Long-Hai Cui, Sang-Yong Son, Ho-Jung Shin, Cheulsu Byun, Hoon Hur, \\ Sang-Uk Han, and Yong Kwan Cho \\ Department of Surgery, Ajou University School of Medicine, Suwon, Republic of Korea \\ Correspondence should be addressed to Sang-Yong Son; spy798@gmail.com
}

Received 18 September 2016; Revised 18 November 2016; Accepted 4 December 2016; Published 9 January 2017

Academic Editor: Haruhiko Sugimura

Copyright ( 92017 Long-Hai Cui et al. This is an open access article distributed under the Creative Commons Attribution License, which permits unrestricted use, distribution, and reproduction in any medium, provided the original work is properly cited.

Background. Although Billroth II (BII) reconstruction is simpler and faster than Billroth I or Roux-en-Y (RY) reconstruction in patients undergoing totally laparoscopic distal gastrectomy (TLDG), BII reconstruction is associated with several complications, including more severe bile reflux. BII Braun anastomosis may be a better alternative to RY reconstruction. Methods. This retrospective study included 56 consecutive patients who underwent TLDG for gastric cancer, followed by BII Braun or RY reconstruction, between January 2013 and December 2015. Surgical outcomes, including length of operation, quantity of blood lost, and postoperative complications, were compared in the two groups. Results. Clinicopathological characteristics did not differ between the BII Braun and RY groups. Mean length of operation was significantly longer in the RY than the BII Braun group (157.3 min versus $134.6 \mathrm{~min}, p<0.010$ ), but length of hospital stay, blood loss, and complication rate did not differ between the two groups. Ileus occurred in three patients (10.0\%) in the RY group. Endoscopic findings 6 months after surgery showed bile reflux in seven $(28 \%)$ patients in the BII Braun group and five $(17.2 \%)$ in the RY group $(p=0.343)$, but no significant differences in rate of gastric residue or degree of gastritis in the remnant stomach in the two groups. Conclusions. B-II Braun anastomosis is a good alternative to RY reconstruction, reducing length of operation and ileus after TLDG.

\section{Introduction}

Gastric cancer is one of the most common cancers and the third leading cause of cancer-related deaths worldwide [1]. Surgical resection remains the only definitive treatment of this malignant disease [2]. Early diagnosis of gastric cancer has resulted in a significant improvement in the long-term survival of patients undergoing surgery [3].

Some patients who undergo surgery for gastric cancer experience postgastrectomy complications, including malabsorption, dumping syndrome, reflux esophagitis, alkaline gastritis, and delayed gastric emptying [4-6]. Reflux gastritis, which occurs mainly after Billroth II (BII) reconstruction, causes long-term distress, impairs patient quality of life, and may lead to increased risk of metachronous cancer development $[7,8]$. The introduction of Roux-en-Y (RY) reconstruction dramatically reduced the rate of alkaline reflux gastritis [9-13]. Early series reported nearly universal success after Roux diversion, resulting in the suggestion that $\mathrm{RY}$ reconstruction be considered a method of primary reconstruction after gastrectomy [14]. However, RY reconstruction has drawbacks, including difficulties performing the procedure and severe complications, such as Roux limb stasis and internal herniation [15-18].

The development of laparoscopic techniques increased the number of patients undergoing totally laparoscopic distal gastrectomy (TLDG) with intracorporeal anastomosis. Use of this surgical method increased the percentage of patients undergoing BII reconstruction, as it is both simple and rapid. BII reconstruction still has limitations, as it can cause more severe bile reflux, increasing the risk of metachronous cancer development $[19,20]$. Braun [21] introduced an enteroenterostomy anastomosis in an attempt to divert food from the afferent limb, thus reducing the incidence of the "vicious 
circle" syndrome. This simple and easy method may be used as a standard method, at least for older patients undergoing TLDG.

It is unclear whether BII Braun anastomosis results in superior perioperative outcomes when compared with RY reconstruction. Few studies to date have compared shortterm outcomes and endoscopic findings after 6 months in patients undergoing laparoscopic intracorporeal anastomosis using these two methods. This study therefore compared the short-term surgical outcomes of BII Braun anastomosis and RY reconstruction in patients who underwent laparoscopic distal gastrectomy (LDG) performed by a single surgeon.

\section{Material and Methods}

2.1. Study Design and Patients. A total of 376 consecutive patients at Ajou University Hospital underwent TLDG by a single surgeon between January 2013 and December 2015. Of these, 167 patients who underwent Billroth I (BI) reconstruction were excluded, as were 153 patients who underwent $\mathrm{BII}$ anastomosis alone without the Braun procedure. Of the remaining 56 patients, 26 underwent BII Braun reconstruction and 30 underwent RY reconstruction. Findings in these two groups were evaluated retrospectively.

The evaluated parameters included patient demographics, comorbidities, operative details, time to first flatus, time to sips of water, length of hospital stay, and postoperative complications. Tumor depth, nodal status, and stage were classified according to the 7th American Joint Committee on Cancer Staging System. Lymph node dissection was performed according to the Guidelines of the Japanese Gastric Cancer Association. All patients underwent follow-up upper gastrointestinal endoscopy 6 months postoperatively. Gastric residue, degree of gastritis, and bile reflux (RGB) classification was analyzed, with higher scores indicating worse symptoms or signs in the remnant stomach [22].

The study was reviewed and approved by the Ajou University Hospital Institutional Review Board.

2.2. Surgical Technique. LDG was performed with the patient in the supine position under general anesthesia. The operator and endoscopist stood on the right side of the patient and the first assistant stood on the left side. The method used one $10 \mathrm{~mm}$ port for the laparoscope, as well as two $12 \mathrm{~mm}$ and two $5 \mathrm{~mm}$ ports. The pneumoperitoneum was maintained between 10 and $13 \mathrm{mmHg}$. Ultrasonically activated shears were used for lymph node dissection.

All patients in our center have undergone reconstruction using intracorporeal anastomosis since April 2010. Following LDG, patients in the BII Braun group underwent gastrojejunostomy about $40 \mathrm{~cm}$ from the ligament of Treitz in antecolic and isoperistaltic manners. Braun anastomosis was performed about $25 \mathrm{~cm}$ distal to the gastrojejunostomy, using a linear stapler $60 \mathrm{~mm}$ in length with white cartilage. Then, entry hole was closed with a $60 \mathrm{~mm}$ long linear staple with white cartilage in tangential direction. RY reconstruction was performed with an antecolic route and isoperistaltic Roux limb (length $30 \mathrm{~cm}$ ) divided $20 \mathrm{~cm}$ from the ligament of Treitz. Side-to-side gastrojejunostomy and side-to-side jejunojejunostomy were performed intracorporeally with a $60 \mathrm{~mm}$ long linear stapler with white cartilage. The entry hole was closed by the same technique of Braun anastomosis. In the Roux-en-Y group, mesenteric defect was routinely repaired by a continuous suture with 3-0 Vicryl (Ethicon, Rome, Italy) or V-Loc 90 (Covidien, Mansfield, Massachusetts), whereas Petersen's defect was not repaired in both groups.

2.3. Statistical Analysis. All statistical analyses were performed using SPSS version 20.0 (SPSS Inc., Chicago, IL, USA). Differences between the two groups were assessed using $\chi^{2}$ tests, Fisher's exact tests, and Student's $t$-tests, as appropriate. A $p$ value $<0.05$ was considered statistically significant.

\section{Results}

Table 1 shows the demographic and clinical characteristics of the two groups. Age, sex, comorbidities, body mass index (BMI), American Society of Anesthesiologists (ASA) score, extent of surgery, number of retrieved lymph nodes, and pathologic stage were similar in the two groups.

Short-term surgical outcomes and postoperative complications are shown in Table 2. Operation time was significantly longer in the RY than in the BII Braun group (157.3 $\pm 33.9 \mathrm{~min}$ versus $134.6 \pm 28.8 \mathrm{~min}, p<0.010)$. Time to first sips of water $(1.8 \pm 0.5$ versus $2.0 \pm 0.9$ days, $p=0.307)$ and length of hospital stay $(7.9 \pm 8.4$ versus $7.0 \pm 1.6$ days, $p=0.583)$, however, did not differ between the two groups. There were also no significant differences in anesthesia time, blood loss, time to first flatus, and postoperative complications. Ileus occurred in three patients $(10.0 \%)$ in the RY group.

Functional outcomes were assessed indirectly by weight change and gastrointestinal symptoms (Table 3). The RY group showed a trend of larger weight loss than the BII Braun group, but body mass index of postoperative 3 and 6 months did not differ between the BII Braun and RY groups (22.2 versus 22.0, $p=0.842$ and 21.9 versus $21.6, p=0.680$ ). Regarding GI symptoms, there was no significant difference in occurrence rate between two groups $(11.5 \%$ in the BII Braun group versus $30.0 \%$ in the RY group, $p=0.114)$. Endoscopic finding was performed at 6 months after surgery in 25 patients (96.1\%) in the BII Braun group and 29 (96.6\%) in the RY group. The grades of gastric residue, remnant gastritis, and bile reflux did not differ in these two groups (Figure 1).

\section{Discussion}

Although the number of LDGs has increased worldwide since its introduction in the 1990s [23], there is a lack of consensus among surgeons regarding the choice of reconstructive procedure after LDG. The three methods, BI, BII, and RY, have advantages and disadvantages. The ideal gastrointestinal reconstruction procedure should minimize postoperative morbidity and improve quality of life.

A survey in Korea in 2009 found that BI was the most frequent type of reconstruction after distal gastrectomy (6581 patients, $63.4 \%$ ), followed by BII reconstruction (3437 
TABLE 1: Clinicopathologic characteristics of the patients.

\begin{tabular}{|c|c|c|c|}
\hline Variable & BII Braun $(n=26)$ & Roux-en-Y $(n=30)$ & $p$ value \\
\hline Age & $60.1 \pm 13.3$ & $57.6 \pm 12.6$ & 0.466 \\
\hline Sex & & & 0.218 \\
\hline Male & $15(57.7 \%)$ & $22(73.3 \%)$ & \\
\hline Female & $11(42.3 \%)$ & $8(26.7 \%)$ & \\
\hline BMI $\left(\mathrm{kg} / \mathrm{m}^{2}\right)$ & $23.3 \pm 3.3$ & $24.0 \pm 3.5$ & 0.491 \\
\hline ASA score & & & 0.453 \\
\hline 1 & $13(50.0 \%)$ & $18(60.0 \%)$ & \\
\hline 2 & $13(50.0 \%)$ & $12(40.0 \%)$ & \\
\hline Comorbidity & $12(46.2 \%)$ & $12(40.0 \%)$ & 0.643 \\
\hline Hypertension & 10 & 6 & \\
\hline Diabetes mellitus & 6 & 7 & \\
\hline Liver diseases & 3 & & \\
\hline Tuberculosis & 2 & 1 & \\
\hline Myocardial Infarction & 1 & & \\
\hline Extent of lymph node dissection & & & 0.906 \\
\hline $\mathrm{D} 1+$ & $10(38.5 \%)$ & $12(40.0 \%)$ & \\
\hline D2 & $16(61.5 \%)$ & $18(60.0 \%)$ & \\
\hline Number of retrieved lymph nodes & $36.7 \pm 15.4$ & $35.8 \pm 10.9$ & 0.793 \\
\hline Pathologic stage & & & 0.043 \\
\hline I & $12(46.2 \%)$ & $23(76.6 \%)$ & \\
\hline II & $7(26.9 \%)$ & $5(16.7 \%)$ & \\
\hline III & $7(26.9 \%)$ & $2(6.7 \%)$ & \\
\hline
\end{tabular}

Values are presented as number (\%) or mean \pm standard deviation.

$\mathrm{BMI}=$ body mass index; ASA = American Society of Anesthesiologists.

TABLE 2: Comparison of surgical outcomes according to the reconstructive procedures.

\begin{tabular}{lccc}
\hline Variable & BII Braun $(n=26)$ & Roux-en-Y $(n=30)$ & $p$ value \\
\hline Operating time (min) & $134.6 \pm 28.8$ & $157.3 \pm 33.9$ & $96.0 \pm 89.8$ \\
Blood loss (ml) & $89.2 \pm 85.5$ & $1.8 \pm 0.5$ & 0.010 \\
Sips of water (d) & $2.0 \pm 0.9$ & $3.1 \pm 0.8$ & $7.9 \pm 8.4$ \\
Soft diet (d) & $3.2 \pm 0.8$ & $6(20.0 \%)$ \\
Hospital stay (d) & $7.0 \pm 1.6$ & & \\
Postoperative complications & $4(15.3 \%)$ & $1(3.3 \%)$ \\
$\quad$ Wound & $1(3.8 \%)$ & $3(10.0 \%)$ \\
$\quad$ Intraluminal bleeding & $1(3.8 \%)$ & $1(3.3 \%)$ \\
$\quad$ Ileus & & $1(3.3 \%)$ \\
$\quad$ Leakage & $1(3.8 \%)$ & \\
$\quad$ Pancreatitis & $1(3.8 \%)$ & $3(10.0 \%)$ \\
$\quad$ Other & $2(7.6 \%)$ & $3(10.0 \%)$ \\
Clavien-Dindo classification & $2(7.6 \%)$ & 0.784 \\
$\quad$ Grades I-II & & \\
$\quad$ Grades III-IV & & \\
\hline
\end{tabular}

Values are presented as number (\%) or mean \pm standard deviation.

patients, 33.1\%), with RY reconstruction rarely performed (332 patients, 3.3\%) [24]. Increased experience with TLDG has increased the use of intracorporeal anastomosis, with KLASS 01 data showing that a significantly larger number of patients underwent BII reconstruction than RY following LDG (232 versus 20, $p<0.001$ ) [25], because BII was both simpler and faster to perform. In Japan, however, the most common method of reconstruction was BI, followed by RY [26]. BII reconstruction was rarely performed by any Japanese surgeons, because it can cause more severe bile reflux, which may strongly correlate with carcinogenesis in the gastric remnant [26]. The results of our study suggest that 
TABLE 3: Comparison of postoperative weight change and gastrointestinal symptoms.

\begin{tabular}{|c|c|c|c|}
\hline Variable & BII Braun $(n=26)$ & Roux-en-Y $(n=30)$ & $p$ value \\
\hline \multicolumn{4}{|c|}{ Length of resected stomach $(\mathrm{cm})$} \\
\hline Lesser curvature & $12.4 \pm 2.6$ & $13.7 \pm 2.6$ & 0.086 \\
\hline Greater curvature & $19.0 \pm 3.9$ & $22.0 \pm 4.9$ & 0.016 \\
\hline \multicolumn{4}{|l|}{ Body weight (kg) } \\
\hline Preoperative & $61.9 \pm 10.7$ & $67.3 \pm 13.1$ & 0.102 \\
\hline Postoperative 3 months & $58.9 \pm 10.3$ & $61.8 \pm 12.0$ & 0.334 \\
\hline Postoperative 6 months & $58.3 \pm 10.3$ & $60.7 \pm 12.2$ & 0.424 \\
\hline \multicolumn{4}{|l|}{ BMI $\left(\mathrm{kg} / \mathrm{m}^{2}\right)$} \\
\hline Preoperative & $23.3 \pm 3.3$ & $24.0 \pm 3.5$ & 0.491 \\
\hline Postoperative 3 months & $22.2 \pm 3.1$ & $22.0 \pm 3.2$ & 0.842 \\
\hline Postoperative 6 months & $21.9 \pm 3.0$ & $21.6 \pm 3.2$ & 0.680 \\
\hline Postoperative GI symptoms & $3(11.5 \%)$ & $9(30.0 \%)$ & 0.114 \\
\hline Diarrhea & 3 & 3 & \\
\hline Constipation & & 2 & \\
\hline Dyspepsia & & 1 & \\
\hline Gas bloating & & 1 & \\
\hline Reflux/soreness & & 2 & \\
\hline
\end{tabular}

Values are presented as number (\%) or mean \pm standard deviation.

$\mathrm{BMI}=$ body mass index; GI = gastrointestinal.

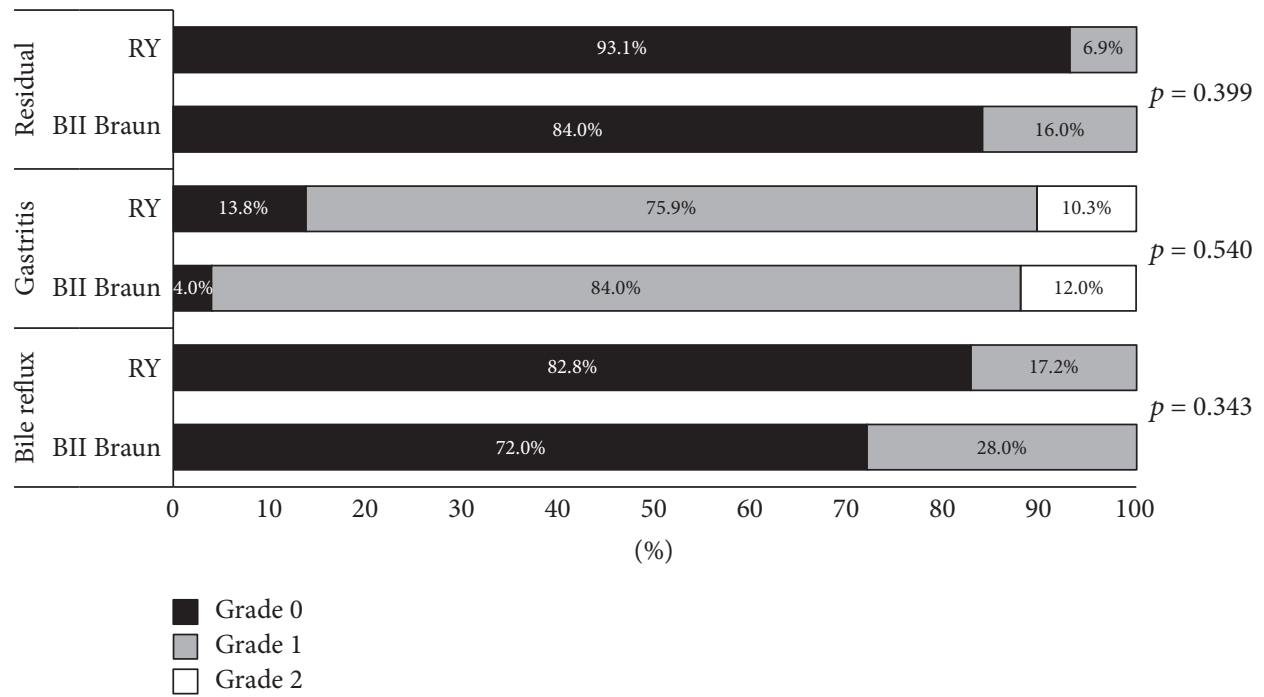

FIGURE 1: RGB score of gastroscopy at postoperative 6 months.

BII Braun reconstruction after LDG for gastric cancer has perioperative outcomes similar to those of RY reconstruction, as shown by rates of postoperative complications and 6month postoperative bile reflux (RGB) scores. These findings indicate that BII Braun anastomosis successfully diverts a substantial amount of bile from the remnant stomach and therefore may be an alternative to RY reconstruction in treating bile reflux.

Postoperative complications leading to malnutrition, such as delayed gastric emptying, anastomotic leak, and dumping syndrome, may require enteral nutritional support, prolong hospital stay, and increase health care costs. A comparison of patients who underwent BII or RY reconstruction found no differences in the rates of postgastrectomy diarrhea (9.1\% versus $9.7 \%$ ), dumping syndrome (6\% versus $3.2 \%)$, and weight gain (78.8\% vs. 90.3\%) [27]. Although our retrospective registry did not include specific information on dumping syndrome and relevant quality-of-life parameters, we evaluated length of hospital stay, discharge destination, and hospital readmission as indirect measures of potential 
postoperative gastrointestinal dysfunction. We found all of these parameters were similar in the BII Braun and RY groups.

A retrospective study from Memorial Sloan Kettering Cancer Center compared outcomes in 122 patients who underwent RY reconstruction and 588 who underwent classic BII reconstruction after pancreaticoduodenectomy [28]. There were no differences in the rates of delayed gastric emptying (10.1\% versus $10.3 \%$ ), reoperation (9.1\% vs. $6.9 \%)$, interventional radiology procedures (9.8\% versus $6.8 \%$ ), length of hospital stay (11 days versus 10 days), and mortality ( $0.9 \%$ versus $2.6 \%)$, findings similar to the results of our study.

Theoretically, LDG with BII Braun anastomosis may also minimize specific complications such as afferent loop syndrome and roux stasis syndrome. Braun anastomosis can divert a substantial amount of bile from the remnant stomach to the efferent loop; thereby it may reduce the afferent loop syndrome compared with BII without Braun anastomosis [29]. In our study, three patients in the RY group experienced ileus, indicating the roux stasis syndrome. Roux stasis syndrome is characterized by symptoms such as nausea, vomiting, epigastric pain, fullness, and difficulties in eating after Roux-en-Y gastrojejunostomy. However, the clinical definition of the roux stasis syndrome is ambiguous, sometimes confusing with postoperative ileus. Thus, more study is required to clarify the reality of roux stasis syndrome.

This study had several limitations, including its retrospective design. It was difficult to identify intraoperative factors that may have influenced the choice of BII Braun or RY reconstruction, but a certain selection bias might influence the present study. For instance, in the size of resected specimen, the mean length of greater curvature is significantly longer in the RY than in the BII Braun group $(22.0 \mathrm{~cm} v s .19 .0 \mathrm{~cm}, p=0.016)$. However, this was not a direct evidence that RY group has larger remnant stomach than BII Braun group and its clinical influence might be limited because the BMI were not different during postoperative 6 months. Moreover, the patient population was relatively small. Furthermore, it is difficult to discern retrospectively whether specific complications were directly related to the type of reconstruction. However, BII Braun anastomosis successfully diverted a substantial amount of bile from the remnant stomach, making this outcome comparable in the two groups.

\section{Conclusions}

The type of reconstruction after LDG had no effect on the rate or distribution of postoperative complications, length of hospital stay, or postoperative bile reflux scores. As BII Braun anastomosis successfully diverted a substantial amount of bile from the remnant stomach, this method may be a good alternative to RY reconstruction in preventing bile reflux. Short-term perioperative outcomes showed that BII Braun anastomosis and RY reconstruction can be considered equally acceptable restorative options following LDG for gastric cancer.

\section{Ethical Approval}

All procedures performed in studies involving human participants were in accordance with the ethical standards of the institutional and/or national research committee and with the 1964 Helsinki declaration and its later amendments or comparable ethical standards. For this type of study formal consent is not required.

\section{Consent}

Informed consent was obtained from all individual participants included in the study.

\section{Disclosure}

The funding source hand no role in the design of this article and will not have any role during its execution or publication.

\section{Competing Interests}

The authors declared no potential conflict of interests relevant to this article.

\section{Authors' Contributions}

Study conception and design were done by Sang-Uk Han, Sang-Yong Son, and Yong Kwan Cho. Acquisition of data was done by Long-Hai Cui. Analysis and interpretation of data were done by Sang-Yong Son and Sang-Uk Han. Drafting of manuscript was done by Long-Hai Cui. Critical revision of manuscript was done by Ho-Jung Shin, Cheulsu Byun, and Hoon Hur.

\section{Acknowledgments}

This study was supported by a grant from the National R \& D Program for Cancer Control, Ministry of Health \& Welfare, Republic of Korea (1320270).

\section{References}

[1] L. A. Torre, F. Bray, R. L. Siegel, J. Ferlay, J. Lortet-Tieulent, and A. Jemal, "Global cancer statistics, 2012," CA Cancer Journal for Clinicians, vol. 65, no. 2, pp. 87-108, 2015.

[2] J.-P. Kim, "Current status of surgical treatment of gastric cancer," Journal of Surgical Oncology, vol. 79, no. 2, pp. 79-80, 2002.

[3] D. H. Roukos, "Current advances and changes in treatment strategy may improve survival and quality of life in patients with potentially curable gastric cancer," Annals of Surgical Oncology, vol. 6, no. 1, pp. 46-56, 1999.

[4] C.-W. Wu, M.-C. Hsieh, S.-S. Lo, W.-Y. Lui, and F.-K. P’Eng, "Quality of life of patients with gastric adenocarcinoma after curative gastrectomy," World Journal of Surgery, vol. 21, no. 7, pp. 777-782, 1997.

[5] R. Tomita, K. Tanjoh, S. Fujisaki, and M. Fukuzawa, "Relation between gastroduodenal interdigestive migrating motor complex and postoperative gastrointestinal symptoms before and after cisapride therapy following distal gastrectomy for early 
gastric cancer," World Journal of Surgery, vol. 24, no. 10, pp. 1250-1257, 2000.

[6] E. R. Woodward and M. P. Hocking, "Postgastrectomy syndromes," The Surgical Clinics of North America, vol. 67, no. 3, pp. 509-520, 1987.

[7] A. C. Fiore, M. A. Malangoni, T. A. Broadie, J. A. Madura, and J. E. Jesseph, "Surgical management of alkaline reflux gastritis," Archives of Surgery, vol. 117, no. 5, pp. 689-694, 1982.

[8] T. C. Northfield and C. N. Hall, "Carcinoma of the gastric stump: risks and pathogenesis," Gut, vol. 31, no. 11, pp. 1217-1219, 1990.

[9] M. K. Bartlett and J. D. Burrington, "Bilious vomiting after gastric surgery. Experience with a modified Roux-Y loop for relief," Archives of Surgery, vol. 97, no. 1, pp. 34-39, 1968.

[10] J. A. van Heerden, J. T. Priestley, G. M. Farrow, and S. F. Phillips, "Postoperative alkaline reflux gastritis. Surgical implications," The American Journal of Surgery, vol. 118, no. 3, pp. 427-433, 1969.

[11] J. A. van Heerden, S. F. Phillips, M. A. Adson, and D. C. Mcllrath, "Postoperative reflux gastritis," The American Journal of Surgery, vol. 129, no. 1, pp. 82-88, 1975.

[12] F. L. Bushkin and E. R. Woodward, "Alkaline reflux gastritis," Major Problems in Clinical Surgery, vol. 20, pp. 48-63, 1976.

[13] T. Kennedy and R. Green, "Roux diversion for bile reflux following gastric surgery," The British Journal of Surgery, vol. 65, no. 5, pp. 323-325, 1978.

[14] J. L. Herrington Jr., "Roux-en-Y diversion as an alternate method of reconstruction of the alimentary tract after primary resection of the stomach," Surgery Gynecology \& Obstetrics, vol. 143, no. 1, pp. 92-93, 1976.

[15] L. H. Karlstrom, N. J. Soper, K. A. Kelly, and S. F. Phillips, "Ectopic jejunal pacemakers and enterogastric reflux after Roux gastrectomy: effect of intestinal pacing," Surgery, vol. 106, no. 3, pp. 486-495, 1989.

[16] L. E. Perino, K. A. Adcock, and J. S. Goff, "Gastrointestinal symptoms, motility, and transit after the Roux-en-Y operation," The American Journal of Gastroenterology, vol. 83, no. 4, pp. 380-385, 1988.

[17] W. D. Harrison, M. P. Hocking, and S. B. Vogel, "Gastric emptying and myoelectric activity following Roux-en-Y gastrojejunostomy," The Journal of Surgical Research, vol. 49, no. 5, pp. 385-389, 1990.

[18] M. P. Hocking, R. G. Carlson, and S. B. Vogel, "Does selective vagotomy prevent delayed gastric emptying and altered myoelectric activity following Roux-en-Y gastrojejunostomy?" The American Journal of Surgery, vol. 163, no. 1, pp. 32-36, 1992.

[19] K. Fukuhara, H. Osugi, N. Takada, M. Takemura, M. Higashino, and H. Kinoshita, "Reconstructive procedure after distal gastrectomy for gastric cancer that best prevents duodenogastroesophageal reflux," World Journal of Surgery, vol. 26, no. 12, pp. 1452-1457, 2002.

[20] Y. Sugiyama, H. Sohma, M. Ozawa et al., "Regurgitant bile acids and mucosal injury of the gastric remnant after partial gastrectomy," The American Journal of Surgery, vol. 153, no. 4, pp. 399403, 1987.

[21] H. Braun, "Ueber die Gastro-enterostomie and Gleichzeutig Ausgefuhrte," Langenbecks Archiv für Chirurgie, vol. 45, no. 2, p. 361, 1893.

[22] M. Kubo, M. Sasako, T. Gotoda et al., "Endoscopic evaluation of the remnant stomach after gastrectomy: proposal for a new classification," Gastric Cancer, vol. 5, no. 2, pp. 83-89, 2002.
[23] S. Kitano, Y. Iso, and K. Sugimachi, "Laparoscopy-assisted billroth I gastrectomy," Surgical Laparoscopy \& Endoscopy, vol. 4, no. 2, pp. 146-148, 1994.

[24] O. Jeong and Y.-K. Park, "Clinicopathological features and surgical treatment of gastric cancer in South Korea: the results of 2009 nationwide survey on surgically treated gastric cancer patients," Journal of Gastric Cancer, vol. 11, no. 2, pp. 69-77, 2011.

[25] W. Kim, H.-H. Kim, S.-U. Han et al., "Decreased morbidity of laparoscopic distal gastrectomy compared with open distal gastrectomy for stage I gastric cancer: short-term outcomes from a multicenter randomized controlled trial (KLASS-01)," Annals of Surgery, vol. 263, no. 1, pp. 28-35, 2016.

[26] K. Kumagai, K. Shimizu, N. Yokoyama, S. Aida, S. Arima, and T. Aikou, "Questionnaire survey regarding the current status and controversial issues concerning reconstruction after gastrectomy in Japan," Surgery Today, vol. 42, no. 5, pp. 411-418, 2012.

[27] A. Csendes, A. M. Burgos, G. Smok, P. Burdiles, I. Braghetto, and J. C. Díaz, "Latest results (12-21 years) of a prospective randomized study comparing billroth ii and roux-en-y anastomosis after a partial gastrectomy plus vagotomy in patients with duodenal ulcers," Annals of Surgery, vol. 249, no. 2, pp. 189-194, 2009.

[28] S. R. Grobmyer, S. T. Hollenbeck, D. P. Jaques et al., "Roux-enY reconstruction after pancreaticoduodenectomy," Archives of Surgery, vol. 143, no. 12, pp. 1184-1188, 2008.

[29] S. B. Vogel, W. E. Drane, and E. R. Woodward, "Clinical and radionuclide evaluation of bile diversion by braun enteroenterostomy: prevention and treatment of alkaline reflux gastritis. An alternative to Roux-en-Y diversion," Annals of Surgery, vol. 219, no. 5, pp. 458-465, 1994. 


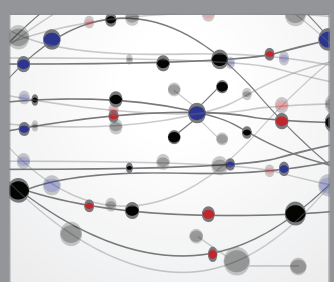

The Scientific World Journal
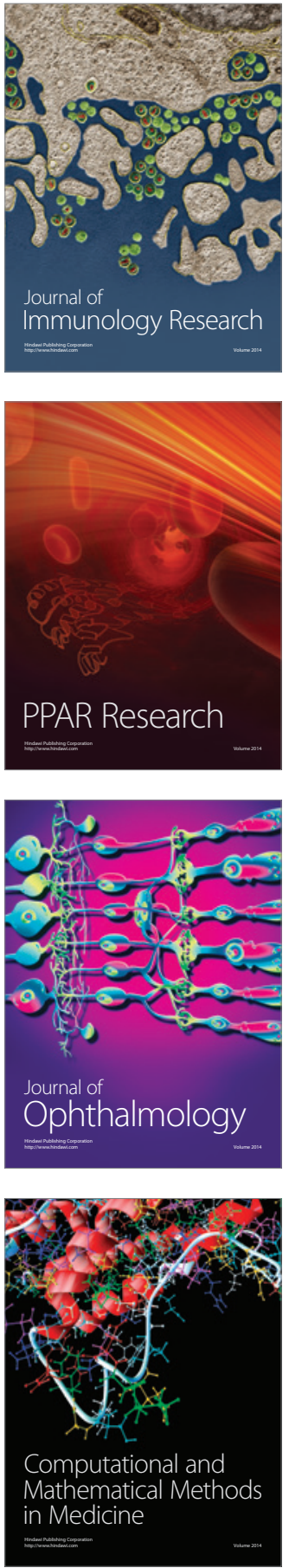

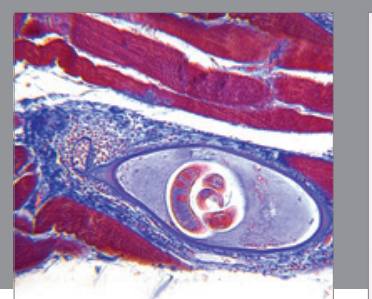

Gastroenterology Research and Practice
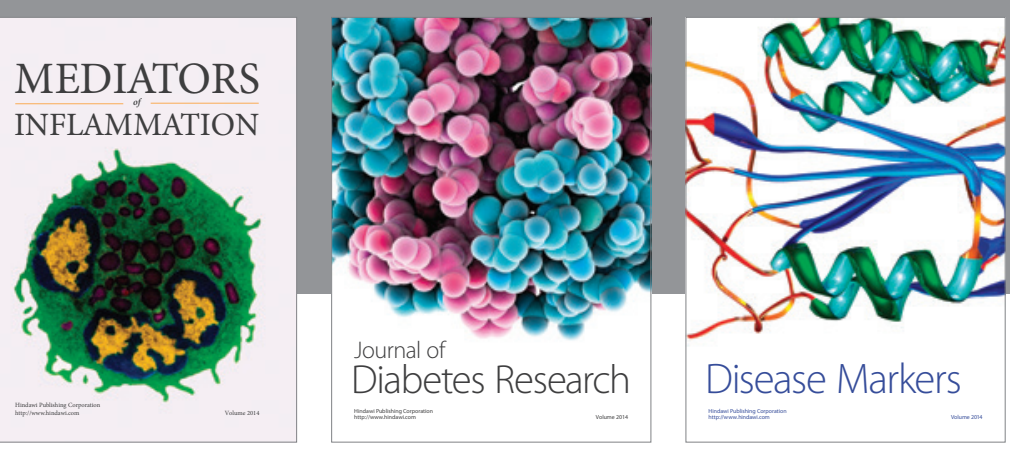

Disease Markers

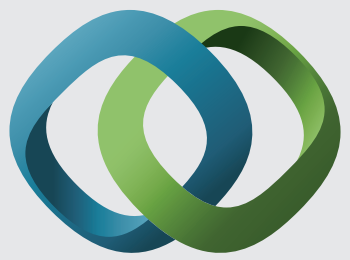

\section{Hindawi}

Submit your manuscripts at

https://www.hindawi.com
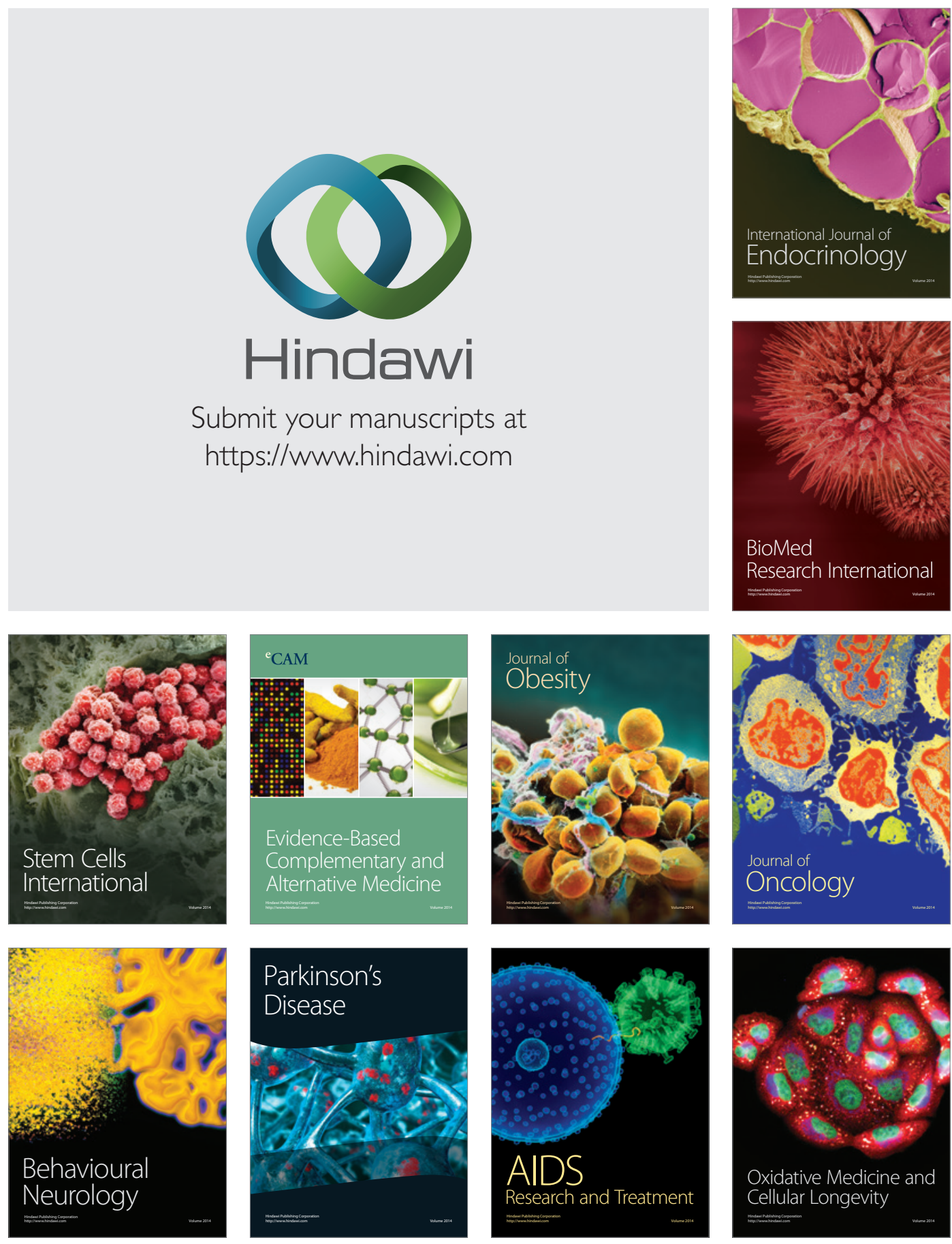